\title{
The Potential Function of KIF17 in Large Yellow Croaker (Larimichthys crocea) Spermatid Remodeling: Molecular Characterization and Expression Pattern During Spermiogenesis
}

\author{
Jingqian Wang \\ Ningbo University \\ Zhao Liu \\ Ningbo University \\ Xinming Gao \\ Ningbo University \\ Chen Du \\ Ningbo University \\ Congcong Hou \\ Ningbo University \\ Daojun Tang \\ Ningbo University
}

\section{Bao Lou}

Zhejiang Academy of Agricultural Sciences

\section{Weiliang Shen}

Ningbo academy of oceanology and fishery

Jun-Quan Zhu ( $\nabla$ zhujunquan@nbu.edu.cn )

Ningbo University https://orcid.org/0000-0001-5908-3777

\section{Research Article}

Keywords: Larimichthys crocea, Spermiogenesis, KIF17, Spermatid remodelinge

Posted Date: June 21st, 2021

DOI: https://doi.org/10.21203/rs.3.rs-612804/v1

License: (c) (1) This work is licensed under a Creative Commons Attribution 4.0 International License. Read Full License 


\section{Abstract}

KIF17, which belongs to the kinesin-2 protein family, plays an indispensable role in mammalian spermiogenesis. However, the role of KIF17 in fish spermatid remodeling during spermiogenesis remains poorly understood. Therefore, we aimed to study the role of KIF17 in spermatid remodeling during Larimichthys crocea (L. crocea) spermiogenesis. The kif17 cDNA sequence, 3247 bp in length, was cloned from $L$. crocea testis, which consisted of a 347 bp 5囚-untranslated region (UTR), 413 bp 3\-UTR, and 2487 bp open reading frame. Bioinformatic analyses revealed that KIF17 obtained from L. crocea ( $L C$-KIF17) exhibited a high sequence identity compared with those from other teleosts and possessed the structural features of other kinesin-2 proteins. Based on structural similarity, we speculate that the role of Lc-KIF17 may be similar to that of KIF17 in other animals. Lc-kif17mRNA was diffusely expressed in $L$. crocea tissues and was highly expressed in the testis, especially at stage IV testicular development. Immunofluorescence analysis revealed that Lc-KIF17 signals colocalized with $\beta$-tubulin signals and migrated from the perinuclear cytoplasm to the side of the nucleus where the tail forms during spermiogenesis. These findings revealed that KIF17 may be involved in L. crocea spermiogenesis. In particular, KIF17 may participate in spermatid remodeling by interacting with perinuclear microtubules during $L$. crocea spermiogenesis. Collectively, this study contributes to an improved understanding of the mechanism underlying $L$. crocea spermiogenesis and provides a basis for further research on $L$. crocea reproduction and development.

\section{Introduction}

Spermiogenesis is a sequential spermatid remodeling, in which round spermatids go through substantial structural and functional changes to differentiate into spermatozoa (Hermo et al. 2010a). Such changes, which include the formation of the acrosome, nucleus shaping, disposal of excess cytoplasm, and generation of the sperm tail contributing to the production of viable sperm and transmission of genetic information to the succeeding generation (Ma et al. 2017). Some of these changes in spermiogenesis rely on the regulation of microtubules, as proven in modified mouse models, which have shown that microtubules are essential for male fertility (O'Donnell and O'Bryan 2014).

Microtubules, an important type of cytoskeleton, consist of a-tubulin and $\beta$-tubulin heterodimers and act as "railways" for protein transport during spermiogenesis (Goodson and Jonasson 2018). Cargo protein transport via microtubules during spermatid remodeling is essential for proper acrosome and sperm tail formation and nuclear shaping. Kinesins are microtubule-associated molecular motor proteins that transport cargo proteins along microtubules using energy derived from ATP hydrolysis (Miki et al. 2005). They are divided into 15 distinct families based on sequence similarity (Lawrence et al. 2004). All kinesin family members share a conserved motor head domain (confers ATPase and microtubule-binding activities) that is linked by a coiled-coil stalk (responsible for dimerization) to a greatly diverged cargobinding tail domain (associated with multiple classes of cargo proteins) (Hirokawa and Takemura 2005). KIF17 is a kinesin-2 family member that mainly exists as a homodimer (KIF17) or a heterotrimer (KIF3A, $\mathrm{KIF} 3 \mathrm{~B} / \mathrm{KIF} 3 \mathrm{C}$, and KAP3). The structural characteristics of KIF17 are consistent with those of other 
kinesin family members (Setou 2000). KIF17 plays an indispensable role in spermiogenesis (Macho 2002; Kimmins et al. 2004; Hogeveen and Sassone-Corsi 2006). Previous studies have demonstrated that KIF17 colocalizes with its cargo protein in the manchette and the principal piece of the sperm tail and participates in mouse spermiogenesis (Saade et al. 2007). There have been studies on the function of KIF17 in mammalian spermiogenesis; however, very few studies have focused on the role of KIF17 in fish spermiogenesis, especially with regarding marine fish.

Large yellow croaker (Larimichthys crocea), a marine fish species belonging to the family Sciaenidae, has a high commercial value in China (Chen et al. 2010). L. crocea undergoes spermatid remodeling, which involves nuclear reshaping, tail formation, and excess cytoplasm removal, during spermiogenesis. It has been used as a model for studying spermiogenesis in fish (Luo et al. 2019; Mu et al. 2019; Zhang et al. 2017). This study aimed to investigate the functions of KIF17 in spermatid remodeling during L. crocea spermiogenesis to contribute to improved understanding of the mechanism underlying fish spermiogenesis and to provide a basis for further research on fish reproduction and development.

\section{Materials And Methods}

Tissue sampling

Tissues (heart, liver, intestine, kidney, brain, muscle, and gill of stage IV L. crocea) and testes (at developmental stages II-V) were collected from from six healthy male $L$. crocea subjects supplied by Gangwan Aquatic Fingerlings Limited Company (Xiangshan, Zhejiang, China).

RNA extraction and cDNA preparation

TRIzol Reagent RNA (Invitrogen, San Diego, CA, USA) was used to extract total RNA from each L. crocea tissue sample. The quantitation and purity of the extracted RNA were determined by agarose gel electrophoresis and nucleic acid protein analyzer. PrimeScript ${ }^{\circledR}$ RT Reagent Kit (Takara, Dalian, China) was used in the quantitative real-time polymerase chain reaction (qRT-PCR) analysis. Smart Rapid Amplification of cDNA Ends (RACE) cDNA Amplification Kit (Clontech, Mountain View, CA, USA) was used to synthesize the first-strand CDNA using 5囚-RACE and 3囚-RACE. All operations were performed according to a previous study (Wang et al. 2019).

Cloning and sequence analyses of kif17cDNA

A full-length kif17 cDNA clone from L. crocea (Lc-kif17) was amplified using degenerate PCR and the RACE approach according to a previous study (Wang et al., 2019). The primers used are listed in Table 1. The PCR products were sequenced through GENEWIZ (Suzhou, China). The deduced amino acid sequence of the KIF17 protein from L. crocea (LC-KIF17) was obtained using the Sequence Manipulation Suite (http://www.bio-soft.net/sms/index.html). The molecular mass (Mw) and theoretical isoelectric point ( $\mathrm{pl}$ ) of Lc-KIF17 were predicted using the Compute $\mathrm{pl} / \mathrm{Mw}$ tool (http://cn.expasy.org/tools/pi_tool.html). The functional domains and tertiary structures of KIF17 
proteins were predicted using the Simple Modular Architecture Research Tool (SMART) (http://smart.embl-heidelberg.de/) and Iterative Threading Assembly Refinement (I-TASSER) (http://zhanglab.ccmb.med.umich.edu/I-TASSER/), respectively. Vector NTI10 software was used to perform multiple protein sequence alignment analyses. Phylogenetic analysis of KIF17 was conducted using MEGA 5.0 software (Wang et al. 2019).

Table 1

Primers and probe sequences used in this study

\begin{tabular}{|c|c|c|}
\hline Primer name & Primer sequence $\left(5^{\prime}-3^{\prime}\right)$ & Purpose \\
\hline KIF17 F1 & CAGGCCTGATHTTYGAYMG & PCR \\
\hline KIF17 F2 & GCCATGATCAGCYTTYTC & PCR \\
\hline KIF17 R1 & ATRGTGGTRGTGMGAYGCTG & PCR \\
\hline KIF17 R2 & CACYTCYTCATTGTADATYTCCA & PCR \\
\hline 3'KIF17 F1 & ATTCTGTACATGCCTTAACA & 3' RACE \\
\hline 3'KIF17 F2 & CGAGCACATCTTTGAGAGTATCC & 3' RACE \\
\hline 3'KIF17 R1 & CGCAGTGACAGTGAGAACATTG & 3' RACE \\
\hline 3'KIF17 R2 & GTCACTTCCCAAAAGGTCTCG & 3' RACE \\
\hline 5'KIF17 F1 & AGGATGTGAGCCCGTTAGATTA & 5' RACE \\
\hline 5'KIF17 F2 & ACCGCTGCCAGTGCTTCAT & 5' RACE \\
\hline 5'KIF17 R1 & СССTCATTATGGATGTAAGATTTGC & 5' RACE \\
\hline 5'KIF17 R2 & TCAGTGACACCCTCAACCAAAG & 5' RACE \\
\hline Lc-kif17-RT-F & TCACTGAGGGATACAATGGCA & qPCR \\
\hline Lc-kif17-RT-F & GTCACTTCCCAAAAGGTCTCG & qPCR \\
\hline$\beta$-actin- $\mathrm{F}$ & СTGTCCCTGTATGCCTCTGGTC & Positive control of qPCR \\
\hline$\beta$-actin-R & CTTGATGTCACGCACGATTTCC & Positive control of qPCR \\
\hline Probe sequence & ATGGTGTAAGACTTCCCGCT & Fluorescence in situ hybridization \\
\hline
\end{tabular}

Quantitative analysis of Lc-kif17 mRNA

The distribution of kif17mRNA in the different tissues of L. crocea and at different stages of testicular development was performed by qRT-PCR as described previously (Wang et al. 2019). The specific primer Lc-kif17- RT-F/R (Table 1) was synthesized by GENEWIZ (Suzhou, China) and was used to amplify the target gene. The $\beta$-actin (Actb) gene was chosen as an internal control.

Fluorescence in situ hybridization (FISH) 
L. crocea testes at stage IV of testicular development were extracted, and frozen testis sections embedded in optimal cutting temperature compound were produced as described previously (Wang et al. 2019). The RNA fluorescence probes of Lc-kif17 for fluorescence in situ hybridization (FISH) were designed using Primer 5.0 software (Table 1) and were conjugated to fluorescein using fluorescein isothiocyanate (FITC) synthesized by GENEWIZ (Suzhou, China). The specificity of the RNA fluorescence probes was evaluated using the Basic Local Alignment Search Tool (BLAST). The experiment was performed according to the methods described by Wang et al. (2019). Finally, the results were photographed and analyzed using a confocal laser-scanning microscope (LSM880; Carl Zeiss, Oberkochen, Germany) and Zen 2009 Light Edition (Carl Zeiss, Oberkochen, Germany), respectively.

Antibodies

Antibodies used for western blotting and immunofluorescence (IF) staining included the following: (1) mouse anti-KIF17 antibody prepared in our laboratory (diluted 1:500 for western blotting and 1:75 for IF staining) (Wang et al. 2019), (2) anti-rabbit glyceraldehyde-3-phosphate dehydrogenase (GAPDH) antibody (diluted 1:1500 for western blotting), (3) horse radish peroxidase (HRP)-conjugated goat antimouse IgG $(\mathrm{H}+\mathrm{L})$ (diluted 1:2000 for western blotting), (4) HRP-conjugated goat anti-rabbit IgG (diluted 1:2000 for western blotting), (5) rabbit anti-tubulin antibody (diluted 1:100 for IF staining), (6) Alexa Fluor 555-labeled goat anti-mouse IgG $(\mathrm{H}+\mathrm{L})$ (diluted 1:500 for IF staining), and (7) Alexa Fluor 488-labeled donkey anti-rabbit IgG $(H+L)$ (diluted 1:500 for IF staining). All antibodies were obtained from Beyotime (China).

Western blotting

The specificity of the mouse anti-KIF17 antibody and tissue distribution of Lc-KIF17 protein were determined by western blotting, as described by Wang et al. (2019) and Liang et al. (2020). Briefly, total protein was extracted using radioimmunoprecipitation assay (RIPA) lysis buffer (Solarbio, Shanghai, China), diluted with $5 \mathrm{X}$ sodium dodecyl sulfate (SDS) sample buffer, and boiled for $10 \mathrm{~min}$. The protein samples were loaded on $8 \%$ polyacrylamide gels, subjected to SDS-polyacrylamide electrophoresis (SDSPAGE), and transferred onto polyvinylidene fluoride (PVDF) membranes (Bio-Rad, Hercules, CA, USA). The PVDF membranes were blocked with $5 \%$ skimmed milk in phosphate buffered saline (PBS) with Tween 20 (PBS-Tween 20) for $2 \mathrm{~h}$ and incubated with anti-KIF17 antibody at $4^{\circ} \mathrm{C}$ overnight. Anti-rabbit GAPDH antibody was used as a control. The membranes were washed with $0.1 \%$ PBS-Tween 20 to remove the excess primary antibody and were incubated with the secondary antibody for $1 \mathrm{~h}$ at $37^{\circ} \mathrm{C}$. The membranes were then washed with $0.1 \%$ PBS-Tween 20 . Finally, the signals were visualized through chemiluminescence imaging (Tanon 5200; Shanghai, China).

Immunofluorescence staining (IF)

IF experiments were performed as described by Wang et al. (2019). After drying at room temperature for $15 \mathrm{~min}$, the frozen testis sections of male $L$. crocea were permeabilized with $0.3 \%$ PBS with Triton X-100 (PBS-Triton X-100) for 20 min, followed by blocking with $5 \%$ bovine serum albumin in $0.1 \%$ PBS-Triton X- 
100 for $1.5 \mathrm{~h}$ and incubation with primary antibody overnight at $4^{\circ} \mathrm{C}$. The frozen sections were washed with $0.1 \%$ PBS-Triton X-100 to remove the excess primary antibody and were incubated with the secondary antibody for $1 \mathrm{~h}$. Excess secondary antibody was removed using $0.1 \%$ PBS-Triton X-100, and diamino-2-phenylindole (DAPI; Beyotime, China) was used to stain the nuclei for $5 \mathrm{~min}$. Finally, the testis sections were mounted with Antifade Mounting Medium (Beyotime, China) and viewed using a confocal laser-scanning microscope (LSM710/780; Carl Zeiss, Oberkochen, Germany).

\section{Results}

Nucleotide and protein sequence analyses

The kif17cDNA sequence of 3247 bp length was cloned from L. crocea testis, which contained a $347 \mathrm{bp}$ 5囚-untranslated region (UTR), a 2487 bp open reading frame (ORF), and a 413 bp 3囚-UTR (Fig. 1). Lc-kif17 encoded a protein composed of 828 amino acids, with a calculated molecular weight of $92.3 \mathrm{kDa}$ and pl of 7.06 .

The functional domains of KIF17 proteins were predicted to be mainly comprised of a conserved motor head domain at amino acid (aa) positions 3 to 343 (3-343 aa), a stalk domain (344-654 aa) containing three coiled-coil regions (395-443 aa, 546-577 aa, and 614-654 aa), and a cargo-binding tail domain (655-844 aa) (Fig. 2a). Using Batch CD-Search, the motor domain (3-343 aa) was determined to be conserved in KIF17 proteins. This domain consists of ATP-hydrolysis sites and microtubule-binding sites and is responsible for the movement of proteins using energy produced from ATP hydrolysis (Harukata et al. 2005). Using I-TASSER, a 3D structural model of Lc-KIF17 was built, showing similarities with the structures of other kinesin-2 family members (Fig. 2b). A model of the putative homodimeric Lc-KIF17 is shown in Fig. 2c.

The deduced motor domain sequence of Lc-KIF17 displayed a high identity with those of KIF17 orthologs from other teleosts, such as Danio rerio (84.5\%), Anabas testudineus (93.8\%), Astatotilapia calliptera (93.5\%), Oncorhynchus tshawytscha (88\%), Oryzias latipes (82.7\%), Mastacembelus armatus (92.4\%), and Larimichthys polyactis (99.1\%) (Fig. 3). Phylogenetic analysis revealed that KIF17 was closely clustered with L. polyactis KIF17 and belonged to a clade with other kinesin-2 family members from other teleosts (Fig. 4).

Tissue expression of LC-KIF17 at mRNA and protein levels

Lc-kif17 mRNA expression in different tissues of L. crocea was detected by qRT-PCR. The results showed that Lc-kif17 mRNA was expressed in the heart, liver, intestines, kidneys, brain, testes, muscles, and gills, with the brain exhibiting the highest expression level, followed by the testis (Fig. 5a). The specificity of the mouse anti-KIF17 polyclonal antibody was evaluated by western blotting, which showed that this polyclonal antibody can be used against Lc-KIF17 (Fig. 5c). Similar to the mRNA expression pattern, the protein expression pattern showed that Lc-KIF17 was highly expressed in the testes (Fig. 5d). 
Expression of the Lc-kif17 mRNA and the Lc-KIF17 protein during stages II-V of testicular development

We further evaluated the expression of Lc-kif17 mRNA during the different stages (II-V) of the development of $L$. crocea testis. The results showed that the Lc-kif17mRNA increased gradually with testicular development, with stage IV showing the highest expression level (Fig. 5b). Similarly, the expression level of the LCKIF17 protein was highest at stage IV (Fig. 5e).

Spatiotemporal expression of the Lc-kif17mRNA during L. crocea spermiogenesis

To examine the spatiotemporal expression of the Lc-kif17 mRNA during the spermiogenesis of $L$. crocea, we designed probes (Table 1) for the Lc-kif17 mRNA using Primer 5.0 and detected the mRNA subcellular localization and expression level via FISH (Fig. 6). Lc-kif17 mRNA signals were distributed in the perinuclear cytoplasm at the early stage of spermiogenesis (Fig. 6a1-a4) and were enhanced and distributed around the nucleus at the middle stage (Fig. 6, b1-b4). At the late stage of spermiogenesis, Lc-kif17 mRNA signals were detected on the side of the nucleus where the tail forms (Fig. 6, c1-c4). In mature sperm, the mRNA signals were mainly distributed in the sperm midpiece (Fig. 6, d1-d4).

Immunofluorescence localization of LC-KIF17 and tubulin during L. crocea spermiogenesis

After the evaluating the spatiotemporal expression of the Lc-kif17 mRNA during L. crocea spermiogenesis, we further studied the spatiotemporal distribution of LCKIF17 and tubulin during spermiogenesis via IF (Fig. 7). The results showed that $L c$-KIF17 colocalized with tubulin during spermiogenesis. Similar to $L c$-kif1 7 mRNA signals, LcKIF17 protein signals were randomly distributed in the perinuclear cytoplasm at the early stage of spermiogenesis (Fig. 7, a1-a4) and were enhanced and distributed around the nucleus at the middle stage (Fig. 7, b1-b4). At the late stage of spermiogenesis, LCKIF17 protein signals were detected on the side of the nucleus where the tail forms (Fig. 7, c1-c4). In mature sperm, the protein signals were mainly distributed in the sperm midpiece (Fig. 7, d1-d4).

\section{Discussion}

KIF17, a kinesin-2 family member, mainly exists as a homodimer (KIF17) or a heterotrimer (KIF3A, $\mathrm{KIF} 3 \mathrm{~B} / \mathrm{KIF} 3 \mathrm{C}$, and KAP3) and plays an indispensable role in spermiogenesis (Hogeveen and SassoneCorsi 2006; Kimmins et al. 2004; Macho 2002; Silverman and Leroux 2009). Currently, there are a few studies on the role of KIF17 in fish spermiogenesis. In the present study, kif17 cDNA was cloned from $L$. crocea, and the amino acid sequence of $L C$-KIF17 was similar to those of other kinesin-2 family members. Characteristic features of members of the kinesin-2 family include a motor head domain, a coiled-coil stalk domain, and a cargo-binding tail domain (Setou 2000). The motor domain confers ATPase and microtubule-binding activities, while the cargo-binding domain interacts with specific cargo proteins. The stalk region, which differs in KIF3A, KIF3B, and KIF3C, contains three coiled-coil domains related to the autoinhibition caused by a folded conformation that suppresses the enzymatic activity of the motor domain in the absence of cargo proteins (Hammond et al. 2010). Multiple alignment analysis revealed that the deduced motor domain sequence of LC-KIF17 displayed a high identity withthat of KIF17 
orthologs from other teleosts. Phylogenetic analysis showed that the deduced amino acid sequence of KIF17 was highly similar to that of other kinesin-2 family members from other teleosts. These results strongly support that LC-KIF17 involves highly conserved sequences, suggesting that its function may also be conserved.

We then detected the distribution of LC-KIF17 at mRNA and protein levels in different tissues of $L$. crocea and at different stages of testicular development. We found that the Lc-kif17mRNA expression pattern was similar to that of $L C-K I F 17$, with the brain showing the highest expression level, followed by the testes. These expression patterns were consistent with those reported in pervious studies (Setou et al. 2000). According to previous studies, the high KIF17 expression level in the brain is likely related to the dendritic transport of neuronal proteins (Chu et al. 2006; Kayadjanian et al. 2007). In the present study, we focused on the high expression level of Lc-KIF17 in the testes, as such an observation may be related to spermiogenesis (Ma et al. 2017). Moreover, the significantly higher expression level of LC-KIF17 at stage IV than at the other stages of testicular development may help delineate the potential functions of $L C$ KIF17 in L. crocea spermiogenesis. Zhang et al. (2016) discovered that stage IV is the most active stage of $L$. crocea spermiogenesis, during which a large number of spermatids are produced.

We also studied the spatiotemporal expression of Lc-kif17 mRNA during spermiogenesis via FISH, and the results showed that $L c-k i f 17$ mRNA signals were continuously expressed throughout different stages of spermiogenesis. Hence, we speculate that based on the high expression levels of Lc-KIF17 at mRNA and protein levels in the testes, especially at stage IV of testicular development, Lc-KIF17 may actively participate in spermiogenesis.

Microtubules are important parts of the cytoskeleton that act as "railways" for cargo protein transport during spermiogenesis (Goodson and Jonasson 2018). Microtubules consist of a-tubulin and $\beta$-tubulin heterodimers arranged in a head-to-tail manner, with the a-tubulin exposed at the minus (slow-growing) end and the $\beta$-tubulin exposed at the plus (fast-growing) end. During spermatid remodeling, the manchette, a typical microtubular-based cargo delivery platform for intramanchette transport (IMT), transiently assembles around and intimately associates with the spermatid nucleus. IMT is believed to be indispensable to nuclear reshaping (Kierszenbaum 2002; Mochida et al. 1998; Soley 1997) and protein delivery to the base of the sperm tail (Kierszenbaum and Tres 2004; Rivkin et al. 1997; Tres and Kierszenbaum 1996). Manchette irregularities in spermatid remodeling induced by specific mutations in or chemical treatment of the $\beta$-tubulin gene results in nuclear abnormalities and multiple coiling of the sperm tail (Meistrich et al. 1990; Meistrich 1993).

The capabilities of microtubules, which are closely associated with the use of kinesins to recruit multisubunit cargo complexes along microtubule tracks during spermatid remodeling, have been studied in mammals. In particular, KIFC5A-like motor proteins have been associated with the manchette and may contribute to spermatid remodeling (Navolanic and Sperry 2000), KIF3A participates in nuclear elongation and tail formation during spermiogenesis by maintaining the structure of the manchette (Lehti et al. 2013), and KIF17 interacts with its cargo protein in the manchette (Saade et al. 2007). In the present 
study, IF results showed that $L c$-KIF17 signals migrated from the perinuclear cytoplasm to the side of the nucleus where the sperm tail forms and eventually accumulated in the sperm midpiece during the development of spermatids into mature sperms. In addition, the dynamic localization of $\beta$-tubulin signals in $L$. crocea showed the overlapping of $\beta$-tubulin signals and LC-KIF17 signals during spermiogenesis, suggesting that a perinuclear microtubular structure involving LC-KIF17 may be involved in spermatid remodeling in $L$. crocea. Based on these results, the predicted functional models of $L c$-KIF17 are shown in Fig. 8.

\section{Conclusions}

In this study, the cDNA of the Lc-kif17gene was cloned from L. crocea. The Lc-KIF17 protein exhibited the typical features of kinesin-2 proteins. Both Lc-kif17 mRNA and Lc-KIF17 protein were highly expressed in L. crocea testis, especially at stage IV of testicular development, and were continuously detected during spermiogenesis, suggesting that KIF17 may play a crucial role in spermiogenesis. Lc-KIF17 signals were found to colocalize with $\beta$-tubulin signals and migrate from the perinuclear cytoplasm to the side of the nucleus where the tail forms during spermiogenesis, revealing the likely involvement of KIF17 in L. crocea spermiogenesis. In particular, KIF17 may participate in spermatid remodeling by interacting with perinuclear microtubules. This study contributes to improved understanding of the mechanism underlying $L$. crocea spermiogenesis and provides evidence for the association of Lc-KIF17 with perinuclear microtubules and the function of $L c$-KIF17 in spermatid remodeling.

\section{Declarations}

\section{Acknowlegements}

This research was funded by the NSFC-Zhejiang Joint Fund for the Integration of Industrialization and Informatization (U1809212), the Scientific and Technical Project of Zhejiang Province (2016C02055-7), the Scientific and Technical Project of Ningbo City (2015C110005), the Collaborative Innovation Center for Zhejiang Marine High-efficiency and Healthy Aquaculture, the K.C. Wong Magna Fund and Scientific Research Fund (XYL19023) in Ningbo University.

\section{Competing interests statement}

No conflict of interest exits in the submission of this manuscript, and manuscript is approved by all authors for publication.

\section{Competing interests}

The authors declare no competing interests.

\section{Availability of data and material}

All data generated or analysed during this study are included in this published article. 


\section{Code availability}

Not applicable

\section{Authors' contributions}

Conceptualization: JQ Wang, Z Liu, CC Hou and JQ Zhu; Methodology: JQ Wang and Z Liu; Formal analysis and investigation: JQ Wang and Z Liu; Writing - original draft preparation: JQ Wang; Writing review and editing: JQ Wang, XM Gao, DJ Tang and C Du; Funding acquisition: JQ Zhu; Resources: B Lou and WL Shen; Supervision: JQ Zhu.

\section{Ethics approval}

Study was approved in advance by the Institutional Animal Care and Use Committee at the Zhejiang Laboratory Animal Research Center and Ningbo University approved the present study.

\section{Consent to participate}

Participate consent has been received from all authors.

\section{Consent for publication}

Permission has been obtained from all authors for this article.

\section{References}

Chen H, Lin GW, Liu ZK, Chen W, Xie YQ, Wang XC (2010) Study on growth characters of cultured Pseudosciaena crocea originated from eastern fujian. Marine Sciences. 34: 1-

5. https://doi.org/10.3724/SP.J.1238.2010.00453

Chu PJ, Rivera JF, Arnold DB (2006) A role for KIF17 in transport of Kv4.2. J. Biol. Chem. 281: 365373. https://doi.org/10.1074/jbc.M508897200

Goodson HV, Jonasson EM (2018) Microtubules and Microtubule-Associated Proteins. Cold Spring Harbor Perspect. Biol. 10. https://doi.org/10.1101/cshperspect.a022608

Hammond JW, Blasius TL, Soppina V, Cai D, Verhey KJ (2010) Autoinhibition of the kinesin-2 motor KIF17 via dual intramolecular mechanisms. J. Cell Biol. 189: 10131025. https://doi.org/10.1083/jcb.201001057

Hermo L, Pelletier RM, Cyr DG, Smith CE (2010a) Surfing the wave, cycle, life history, and genes/proteins expressed by testicular germ cells. Part 2: changes in spermatid organelles associated with development of spermatozoa. Microsc. Res. Tech. 73: 279-319. https://doi.org/10.1002/jemt.20787 
Hirokawa N, Takemura R (2005) Molecular motors and mechanisms of directional transport in neurons. Nat. Rev. Neurosci. 6: 201-214. https://doi.org/10.2169/internalmedicine.37.65

Hogeveen KN, Sassone-Corsi P (2006) Regulation of gene expression in post-meiotic male germ cells: CREM-signalling pathways and male fertility. Hum. Fertil. 9: 73-

79. https://doi.org/10.1080/14647270500463400

Kayadjanian N, Lee HS, Pina-Crespo J, Heinemann SF (2007) Localization of glutamate receptors to distal dendrites depends on subunit composition and the kinesin motor protein KIF17. Mol. Cell. Neurosci. 34: 219-230. https://doi.org/10.1016/j.mcn.2006.11.001

Kierszenbaum AL (2002) Intramanchette transport (IMT): managing the making of the spermatid head, centrosome and tail. Mol. Reprod. Dev. 63: 1-4. https://doi.org/10.1002/mrd.10179

Kierszenbaum AL, Tres LL (2004) The acrosome-acroplaxome-manchette complex and the shaping of the spermatid head. Arch. Histol. Cytol. 67: 271-284. https://doi.org/10.1679/aohc.67.271

Kimmins S, Kotaja N, Fienga G, Kolthur US, Brancorsini S, Hogeveen K, Monaco L, Sassone-Corsi P (2004) A specific programme of gene transcription in male germ cells. Reprod. BioMed. Online. 8: 496500. https://doi.org/10.1016/S1472-6483(10)61094-2

Lawrence CJ, Dawe RK, Christie KR, Cleveland DW, Dawson SC, Endow SA, Goldstein LSB, Goodson HV, Hirokawa N, Howard J, Malmberg RL, McIntosh JR, Miki H, Mitchison TJ, Okada Y, Reddy ASN, Saxton WM, Schliwa M, Scholey JM, Vale RD, Walzak CE, Wordeman LA (2004) A standardized kinesin nomenclature. J. Cell. Biol. 167: 19-22. https://doi.org/10.1083/jcb.200408113

Lehti MS, Kotaja N, Sironen A (2013) KIF3A is essential for sperm tail formation and manchette function. Mol. Cell. Endocrinol. 377: 44-55. https://doi.org/10.1016/j.mce.2013.06.030

Liang YJ, Ni JH, Wang LM, Tan FQ, Yang WX (2020) KIF3A regulates the Wnt/ $\beta$-catenin pathway via transporting $\beta$-catenin during spermatogenesis in Eriocheir sinensis. Cell Tissue Res.

381. https://doi.org/10.1007/s00441-020-03220-x

Luo SY, Gao XM, Ding J, Liu C, Du C, Hou CC, Zhu JQ, Lou B (2019) Transcriptome sequencing reveals the traits of spermatogenesis and testicular development in large yellow croaker (Larimichthys crocea). Genes. 10. https://doi.org/10.3390/genes10120958

Ma DD, Wang DH, Yang WX (2017) Kinesins in spermatogenesis. Biol. Reprod. 96: 267276. https://doi.org/10.1095/biolreprod.116.144113

Macho B (2002) CREM-Dependent transcription in male germ cells controlled by a kinesin. Science. 298 : 2388-2390. https://doi.org/10.1126/science.1077265 
Meistrich ML (1993) Nuclear Morphogenesis during Spermiogenesis-ScienceDirect. Molecular Biology of the Male Reproductive System. https://doi.org/10.1016/B978-0-08-091764-1.50007-6

Meistrich ML, Trostle-Weige PK, Russell LD (1990) Abnormal manchette development in spermatids of azh/azh mutant mice. Am. J. Anat. 188: 74-86. https://doi.org/10.1002/aja.1001880109

Miki H, Okada Y, Hirokawa N (2005) Analysis of the kinesin superfamily: insights into structure and function. Trends Cell Biol. 15: 467-476. https://doi.org/10.1016/j.tcb.2005.07.006

Mochida K, Tres LL, Kierszenbaum AL (1998) Isolation of the rat spermatid manchette and its perinuclear ring. Dev. Biol. 200: 46-56. https://doi.org/10.1006/dbio.1998.8942

Mu DI, Du C, Fu SY, Wang JQ, Hou CC, Tang DJ, Zhu JQ (2019) Molecular Characterization, Tissue Distribution and Localization of Larimichthys crocea Kif3a and Kif3b and Expression Analysis of Their Genes During Spermiogenesis. J. Ocean Univ. China. 18: 14511469. https://doi.org/10.1007/s11802-019-3987-2

Navolanic PM, Sperry AO (2000) Identification of isoforms of a mitoticmotor in mammalian spermatogenesis. Biol. Reprod. 62: 1360-1369. https://doi.org/10.1095/biolreprod62.5.1360

O’Donnell L, O’Bryan MK (2014) Microtubules and spermatogenesis. Semin. Cell Dev. Biol. 30: 4554. https://doi.org/10.1016/j.semcdb.2014.01.003

Rivkin E, Cullinan E, Tres L, Kierszenbaum A (1997) A protein associated with the manchette during rat spermiogenesis is encoded by a gene of the TBP-1-like subfamily with highly conserved ATPase and protease domains. Mol. Reprod. Dev. 48: 77-89. https://doi.org/10.1002/(SICl)10982795(199709)48:1<77::AID-MRD10>3.0.C0;2-T

Saade M, Irla M, Govin J, Victorero G, Samson M, Nguyen C (2007) Dynamic distribution of spatial during mouse spermatogenesis and its interaction with the kinesin KIF17b. Exp. Cell Res. 313: 614626. https://doi.org/10.1016/j.yexcr.2006.11.011

Setou M (2000) Kinesin Superfamily Motor Protein KIF17 and mLin-10 in NMDA Receptor-Containing Vesicle Transport. Science. 288: 1796-802. https://doi.org/10.1126/science.288.5472.1796

Silverman MA, Leroux MR (2009) Intraflagellar transport and the generation of dynamic, structurally and functionally diverse cilia. Trends Cell Biol. 19: 306-316. https://doi.org/10.1016/j.tcb.2009.04.002

Soley J (1997) Nuclear morphogenesis and the role of the manchette during spermiogenesis in the ostrich (Struthio camelus). J. Anat. 190: 563-576. https://doi.org/10.1046/j.1469-7580.1997.19040563.x

Tres L, Kierszenbaum A (1996) Sak57, an acidic keratin initially present in the spermatid manchette before becoming a component of paraaxonemal structures of the developing tail. Mol. Reprod. Dev. 44: 395-407. https://doi.org/10.1002/(SICI)1098-2795(199607)44:33.0.C0;2-\# 
Wang JQ, Gao XM, Zheng XB, Hou CC, Xie QP, Lou B, Zhu JQ (2019) Expression and potential functions of kif3a/3b to promote nuclear reshaping and tail formation during Larimichthys polyactis spermiogenesis. Dev. Genes Evol. 229: 161-181. https://doi.org/10.1007/s00427-019-00637-5

Zhang B, Xue D, Wang J, Li Y, Liu J (2016) Development and preliminary evaluation of a genomewide single nucleotide polymorphisms resource generated by rad-seq for the small yellow croaker (Larimichthys polyactis). Mol Ecol Resour. 16: 755-768. https://doi.org/10.1111/1755-0998.12476

Zhang DD, Gao XM, Zhao YQ, Hou CC, Zhu JQ (2017) The C-terminal kinesin motor KIFC1 may participate in nuclear reshaping and flagellum formation during spermiogenesis of Larimichthys crocea. Fish Physiol. Biochem. 43: 1351-1371. https://doi.org/10.1007/s10695-017-0377-9

\section{Figures}


1 acatggggaacttggaaacgccgtgagaagagtagcaggagacctgtaaggacacctcgcgaagactaacaccaccgtgtctggacatatttccgccttccttca

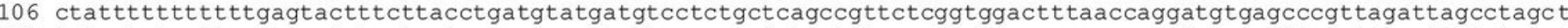

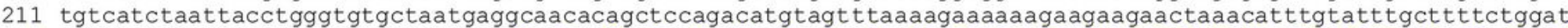

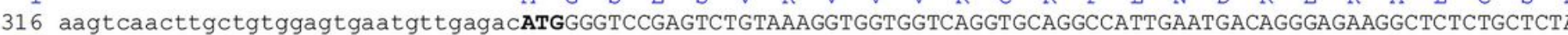

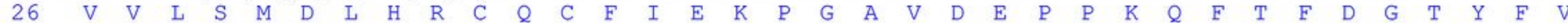
421 AGGTGGTGCTGTCCATGGACCTGCACCGCTGCCAGTGCTTCATAGAGAAGCCCGGGGCGGTGGATGAACCACCCAAGCAGTTCACCTTTGACGGGACCTACTTTG

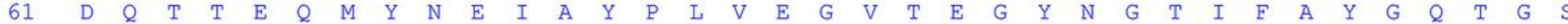
526 TTGATCAAACCACTGAGCAGATGTATAATGAGATTGCCTATCCTTTGGTTGAGGGTGTCACTGAGGGATACAATGGCACAATTTT TGCCTATGGACAAACTGGAA

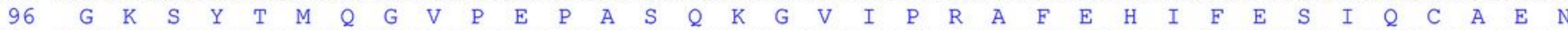
631 GCGGGAAGTCTTACACCATGCAGGGGGTGCCTGAGCCTGCATCCCAGAAGGGGGTTATTCCACGAGCCTTCGAGCACATCTTTGAGAGTATCCAGTGTGCAGAAA

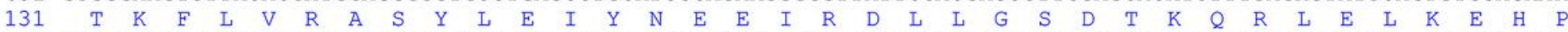
736 ATACAAAATTCCTAGTGAGGGCCTCCTACTTGGAGATTTATAATGAAGAAATCCGAGACCTTTTGGGAAGTGACACCAAACAGAGATTGGAGCTGAAAGAGCATC

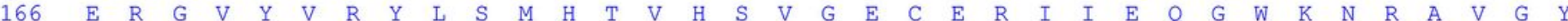
841 CAGAGCGCGGCGTGTATGTGCGGTACCTCTCCATGCACACTGTGCACAGTGTGGGGGAGTGCGAGAGAATCATAGAGCAAGGATGGAAGAACAGGGCAGTGGGCT

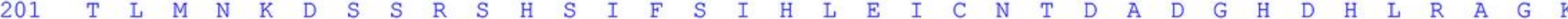
946 ACACACTGATGAACAAAGACTCCTCTCGCTCACACTCTATCTTCAGCATCCATTTGGAGATCTGCAACACAGATGCAGATGGCCATGATCATCTCAGAGCAGGTA

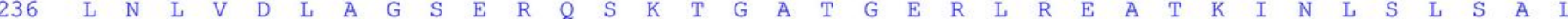
1051 AACTCAACCTTGTTGACCTGGCAGGAAGCGAGCGTCAGTCTAAAACCGGTGCAACTGGTGAGCGACTCCGCGAGGCCACCAAGATCAACCTGTCCCTGTCTGCCC $\begin{array}{llllllllllllllllllllllllllllllllllllllll}271 & G & N & V & I & S & A & L & V & D & G & R & S & K & Y & I & P & Y & R & D & S & K & L & T & R & L & L & Q & D & S & L & G & G & N & T & R\end{array}$ 1156 TGGGGAACGTCATCTCTGCCCTGGTGGACGGACGCTCCAAATACATCCCCTATCGGGACTCCAAGCTCACCAGACTGCTGCAGGACTCTCTGGGAGGAAACACAC $\begin{array}{llllllllllllllllllllllllllllllllllllllllllllll}306 & T & L & M & I & A & C & L & S & P & A & D & N & N & Y & E & E & T & L & S & T & L & R & Y & A & N & R & A & K & S & I & Q & N & R & P & R\end{array}$ 1261 GCACCTTGATGATCGCCTGTCTCTCCCCAGCAGACAATAACTACGAGGAAACCCTGAGCACGCTGCGATATGCCAACCGAGCCAAGAGCATCCAGAACAGGCCTC

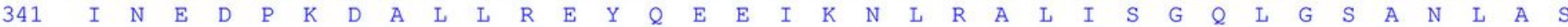
1366 GCATCAATGAGGACCCCAAGGATGCTCTGCTCCGAGAGTATCAGGAGGAGATTAAGAATTTGCGAGCCCTGATCTCAGGCCAGCTGGGCTCTGCTAACCTTGCAT

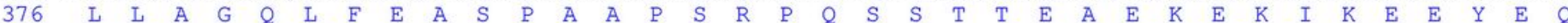

1471 CTCTGCTGGCTGGTCAGTTGTTTGAAGCATCCCCAGCTGCTCCTTCAAGGCCGCAGTCTAGCACCACAGAAGCAGAGAAGGAGAAGATTAAAGAGGAGTACGAAC

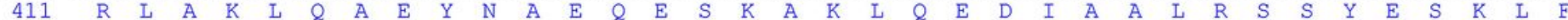
1576 AGAGGCTGGCCAAATTGCAGGCTGAGTACAATGCGGAGCAGGAGTCCAAAGCAAAGCTGCAGGAGGATATTGCTGCACTGCGTTCCTCA TATGAATCCAAGCTGT

$\begin{array}{llllllllllllllllllllllllllllllllllllllllll}446 & D & L & E & K & A & R & A & S & R & G & S & S & V & L & K & N & G & S & G & K & S & T & A & H & N & K & E & A & S & S & V & S & S & S & C\end{array}$

1681 TTGATCTGGAGAAGGCACGAGCCAGCAGGGGGAGCTCTGTCCTAAAGAATGGTAGTGGAAAATCAACTGCCCACAACAAAGAAGCATCATCAGTGAGCTCTAGC T

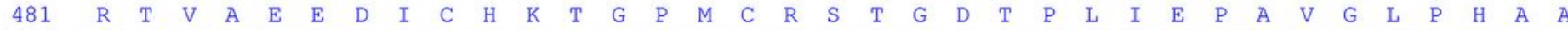

1786 GTAGGACAGTCGCTGAGGAAGACATCTGCCACAAAACTGGCCCTATGTGCCGCAGTACTGGAGACACTCCCCTGATCGAGCCTGCTGTAGGCCTACCTCATGCAG

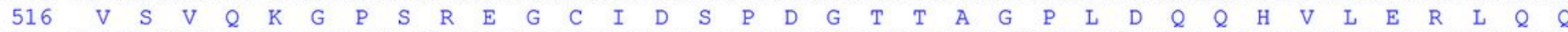
1891 CAGTCAGTGTTCAGAAAGGTCCAAGCAGAGAGGGGTGCATAGACTCACCTGACGGCACCACTGCAGGGCCTCTGGACCAACAACATGTCCTGGAGAGACTGCAGC

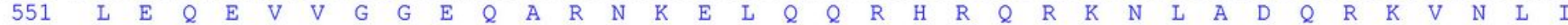

1996 AGCTGGAACAGGAGGTGGTGGGAGGAGAACAGGCCAGGAACAAAGAGTTACAGCAGAGACACCGGCAGAGGAAGAACCTCGCTGACCAGAGAAAAGTTAATCTAA

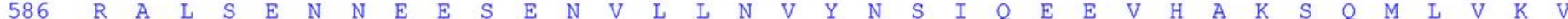
2101 TCCGCGCTCTATCAGAGAACAATGAAGAGAGTGAAAATGTTCTGTTGAATGTCTACAACTCCATCCAGGAGGAGGTCCATGCCAAAAGCCAGATGTTGGTCAAGG

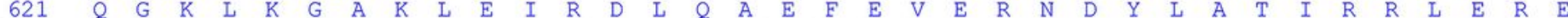
2206 TCCAGGGCAAGCTGAAAGGAGCCAAACTGGAGATCCGTGACCTGCAGGCAGAGTTCGAGGTGGAGAGGAATGACTACCTGGCAACCATCCGGCGGCTGGAGCGAG

$\begin{array}{lllllllllllllllllllllllllllllllllllllll}656 & G & Q & L & L & H & S & L & L & E & R & M & V & P & L & V & R & R & D & C & N & Y & S & N & L & D & R & L & K & K & E & A & V & W & D & B\end{array}$

2311 AGGGCCAGTTACTGCACAGCCTGCTCGAGCGCATGGTGCCCCTGGTGCGCCGTGACTGCAACTACAGCAACCTGGACCGATTGAAAAAAGAAGCTGTTTGGGACG

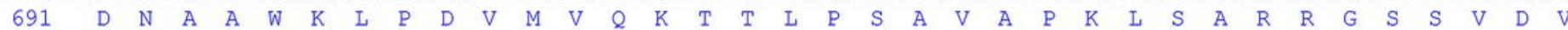
2416 AGGACAACGCAGCCTGGAAGCTGCCGGATGTGATGGTGCAGAAAACAACGTTGCCTTCAGCAGTGGCTCCAAAACTTTCAGCTCGCAGAGGTTCATCTGTTGATG

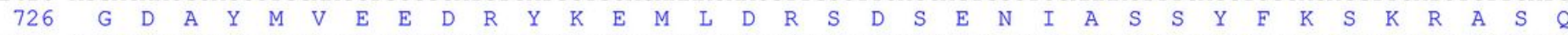
2521 TTGGGGATGCATACATGGTGGAGGAGGACAGGTACAAGGAAATGTTAGACCGCAGTGACAGTGAGAACATTGCTAGCAGCTACTTCAAGTCAAAGAGAGCGAGCC

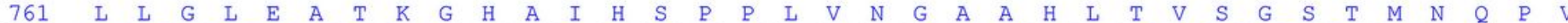
2626 AACTGCTGGGACTTGAAGCTACCAAAGGACACGCCATCCACTCTCCTCCCCTGGTGAACGGGGCAGCCCACCTCACTGTGAGCGGTTCCACCA TGAACCAACCTG

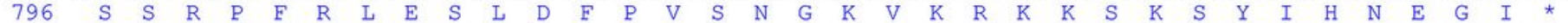
2731 TGAGCTCGCGCCCCTTCCGCCTGGAGTCGTTGGATTTCCCGGTGTCCAATGGTAAGGTGAAGCGCAAAAAAAGCAAATCTTACATCCATAATGAGGGGATT TGAa 2836 aagactgtgaattccctacatgccttaacacatcatcacattgtccgctgccttttgacattgatcgcatagtgaatattaaatattgaagttttgataaccact 2941 gctaaccagaaatgtatataattagcacttttttcatgtgttttaaatctcattttgaatgctcacttgaacaaatgctaccttaagtatgatactaccctt 3046 gaattctttcctatacctaaaacatgtgatttgtgcactgtgagactcaaagtccagctactgtaatatttaccttattatgcatcttaatgttgctgattgta 3151 aaccagattttaacctgttactttactaacatttaaactccaccgttgctgctgaatagagcaaataaattatttatccacttaaaaaaaaa

\section{Figure 1}

The full-length cDNA of the Lc-kif17 gene, which was 3247 bp long, consisted of a 347 bp 5囚-untranslated region (UTR), a 413 bp open reading frame (ORF), and a 413 bp 3凶-UTR. The initiation codon (ATG) and the termination codon (TGA) are in boldface 
$\mathbf{a}$

Head

Stalk

Tail

b

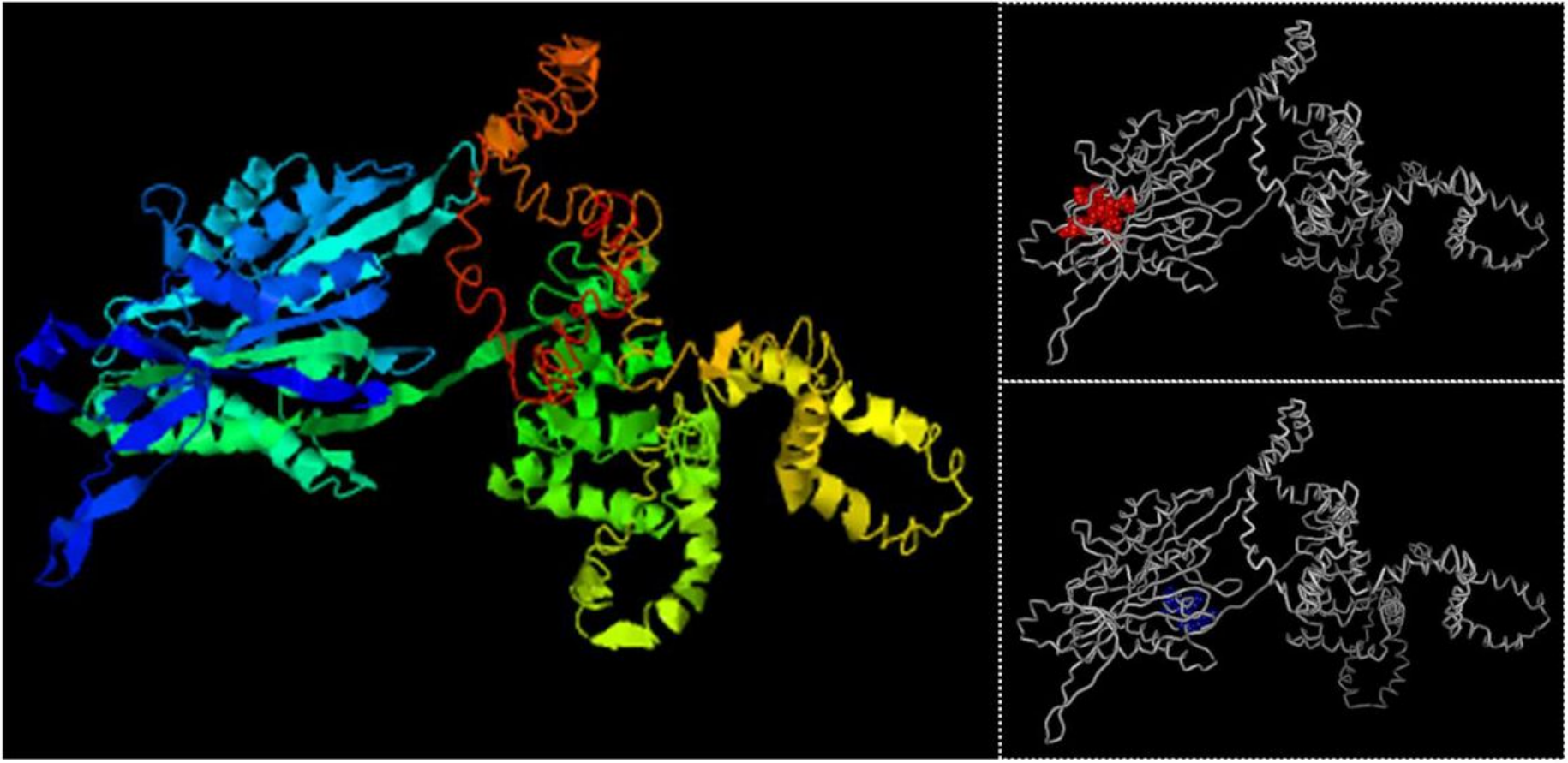

c

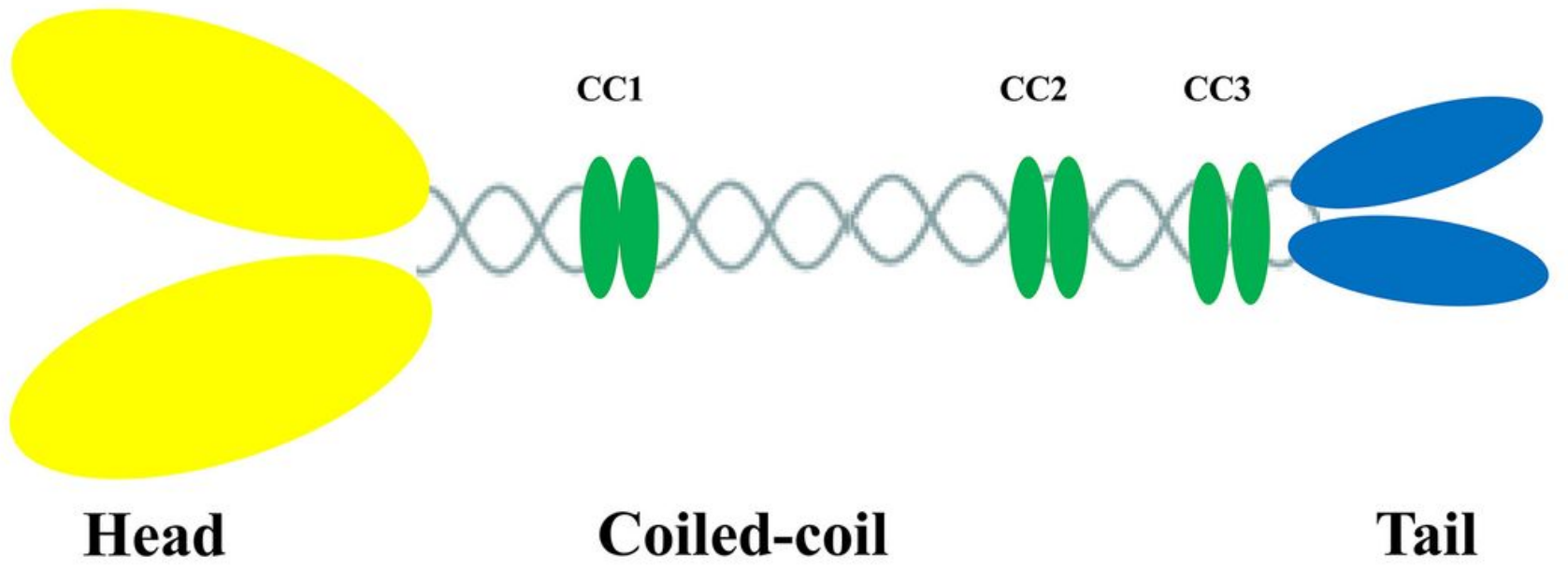

Figure 2

Lc-KIF17 protein structure. (a) Protein structural domain of Lc-KIF17. (b) A 3D structural model of LcKIF17. Red and blue balls represent the ATP-hydrolysis sites and microtubule-binding sites, respectively. (c) A model of the putative Lc-KIF17 homodimeric complex 
Larimichthys crocea (1) SESVKVVVRCAPLNDREKALCSKVVLSMDLHRCQCFIEKPGAVDEPPKQFTFDGTYFVDQTTEQMYNEIAYPLVEGVTEGYNGTIFAVGQ Danio rerio (1) SESVKVVVRCRPLNNREKAMNCKIVVSVDSTHCQCFIKKPGDTEEPPKQFTFDGTYYINHSTEEVYNEIAYPLVEGVTEGYNGT IFAYGQ Anabas testudineus (1) SESVKVVVRdRPLNREKALGSKMALSMDLHRCQCFIEKPGAADEPPKQFTFDGTYFIDQTTEQMYNEIAYPLVEGVTEGYNGTIFA)dQ Astatotilapia calliptera (1) SESVKVVVRCAPLNDREKALSCKMVLTMDLQRCQCFIEKPGAVDEPPKQFTFDGTYYIDQTTEQMYNEIAYPLVEGVTEGYNGTIFAYGQ Oncorhynchus tshawytscha (1) SEAVKVVVRCRPMNDREQILNCKMVVSVETSRCQCLIKKPGAKEEPPKQFTFDGTYFIGQTTEQMYNEIAYPLVEGVTEGYNGTIFA) IQ Oryzias latipes (1) SESVRVVRRQPLNDREKAESPEAVLSVEPQLCQCSIRRPGGGDPPKQFTFDGAYSGEHSTERL YNDTVCPLVEGVTEGYNGT IFAYGQ Mastacembelus armatus (1) SECVKVVVRCAPLNDREKALGSKMVLSMDLHLCQCFIEKPGVADEPPKQFTFDGTYFIEQSTEDMYNEIAYPLVEGVTEGYNGTIFAYdQ Larimichthys polyactis (1) SESVKVVVRdPPLNDREKALCSKVVLSMDLHRCQCFIEKPGÄVDEPPKQFTFDGTYFVDQTTEQWYNEIAYPLVEGVTEGYNGTIFA) CQ Consensus (1) SESVKVVVRQRPLNDREKAL SKMVLSMDLHRCQCFIEKPGA DEPPKQFTFDGTYFIDQTTEQMYNEIAYPLVEGVTEGYNGTIFAYGQ 91

Larimichthys crocea (91) TđGGKSYTMQGVPEPASQKKGVIPRAFEHIFFSIQCAENTKFLVRASYLEIYNEEIRDLLGSDTKQRLELKEHPERGVYVRYLSMHTVHSV Danio rerio (91) TGQGKSH TMQGVPDPPSQRGIIPRAFEHIFETIQCAENTKFLVRASYLEIYKEEIRDLLGKDIKQKMELKEHPERGVYVRDLTMQTVHSV

Anabas testudineus (91) IdGGKSHTMQGLSDPASQRGVIPRAFEHIFESIQCAENTKFLVRASYLEIYNEEIRDLLGNNTKQRLELKEHPERGVYVQYLSMHTVHSV Astatotilapia calliptera (91) IdSGKSHTMQGVSEPATQKGVIPRAFEHIFESIQCAENTKFLVRASYLEIYNEEIRDLLGSD̄KAKQRLELKEHPERGVYVRDLSMHTVHSV Oncorhynchus tshawytscha (91) IddGKSH TMQGVSEPAAQRGVIPRAFEHIFESIQCAENTKFLVRASYLEIYNEEIRDLLGNDTKQKMELKEHPEHGVYVRDLSLHTVHSV Oryzias latipes (91) TIGSGKSPTMQGVSEPEAQRGVIPRAFEHVFETIQCAENTKFLVRACFLEIYNEDIRDLLGNDTKQRLELKEHPERGVYVRDLSMHTVHGV IdSGKSHTMQGVSEPATQRGVIPRAFEHVFESIQCAENTKFLVRASYLEIYNEEIRDLLGNDTKQRLELKEHPERGVYVRDLSMHTVHTV IdSGKS YTMQGVPEPASQKGVIPRAFEHIFESIQCAENTKFLVRASYLEIYNEEIRDLLGSDTKQRLELKEHPERGVYVRYLSMHTVHSV 181

Larimichthys crocea (181) GECERIIEQGWKNRAVGYTLMNKDSSRSHSIFSIHLEICNTDÄDGHDHLRAGKLNLVRLAGSERQSKTGATGERLREATKINLSLSALGN Danio rerio (181) GECERVMDLGWRNRSVGYLMNKDSSRSHSIFTIHLEICSIDTAGDEHLRAGKLNLVDLAGSERQSKTGATGDRLQEATKINLSLSALGN

Anabas testudineus (181) GECERIILQGWKNRAVAYTLMNKDSSRSHS IFSIHLEICSTDEAGQDHLRAGKLNLVDLAGSERQSKTGATGERLREATKINLSLSALGN Astatotilapia calliptera (181) GECERIIEKGWRNRAVGYTLMNKDSSRSHS IFTIHLEICSTDSSDQDHLRAGKLNLVDLAGSERQSKTGATGERLREATKINLSLSALGN Oncorhynchus tshawytscha (181) GECERIMVQGWGNRSVGYTLMNKDSSRSHS IFTIHMEICNTDSAGEDHLRAGKLNLVDLAGSERQSKTGATGERLREATKINLSLSALGN Oryzias latipes (181) GECERMMERGCRNRAVGSTLMNKDSSRSHS IFTLHLEICRTDSSGRDHLRAGKLNLYDLAGSERQSKTGATGERLREATKINLSLSALGN Mastacembelus armatus (181) GECERIIEQGIRNRAVGYTLMNKDSSRSHSIFTIHLEICNTDAGGQVHLRAGKLNLV ILAGSERQSKTGASGERLREATKINLSLSALGN Larimichthys polyact is (181) GECERIIEQGWKNRAVGYTLMNKDSSRSHSIFSIHLEICNTDADGHD̈HLRAGKLNLY LLAGSERQSKTGATGERLREATKINLSLSALGN Consensus (181) GECERIIEQGWRNRAVGYTLMNKDSSRSHSIFTIHLEICNTDAAG DHLRAGKLNLVDLAGSERQSKTGATGERLREATKINLSLSALGN 271 341

Larimichthys crocea (271) VISALVDGRSKYIPYPDSRLLTRLLDSLGGNTRTLMIIACLSPADNNYEETLSTLRYANRAKSIQNRPRINE Danio rerio (271) VISALVDGRSKYIPYPDSYLLTPLLQDSLGGNTRTLMVACLSPADNNYEESISTLRYANRAKS IQNRPRINE

Anabas testudineus (271) VISALVDGRSKYIPIPDSKLTRLLQDSLGGNTRTLMIACLSPADNNYEESLSTLRYANRAKS IQNRPRVNE Astatotilapia calliptera (271) VISALVDGRSRYIPY RDSHLIFLLQDSLGGNTRTLMIACLSPADNNYEESLSTLRYANRAKSIQNRPRINE Oncorhynchus tshawytscha (271) VISALVDGRSKHIP)|FDSKLI | LLLQDSLGGNTRTLMVACLSPADNNYEESLSTLRYANRAKNIQNRPRINE Oryzias latipes (271) VISALVDGRSKYIPY|RDSKLLTRLLQDSLGGNTRTLMIACLSPAESNYEESLSTLRYANRAKSIQNRPRVNE Mastacembelus armatus (271) VISALVDGRSKYIPY BDSKLLIRLLQDSLGGSTRTLMIACLSPADNNYEESLSTLRYANRAKSIQNRPRINE Larimichthys polyactis (271) VISALVDGRSKYIPI FDSKLIPLLQDSLGGNTRTLMIACLSPADNNYEETLSTLRYANRAKSIQNRPRINE Consensus (271) VISALVDGRSKY IPYRDSKLLTRLLQDSLGGNTRTLMIACLSPADNNYEESLSTLRYANRAKSIQNRPRINE

\section{Figure 3}

Comparison of the motor domain sequences of KIF17 orthologs. Amino acid sequences of KIF17 orthologs from Danio rerio (XP_001919146.1), Anabas testudineus (XP_026204326.1), Astatotilapia calliptera (XP_026022883.1), Oncorhynchus tshawytscha (XP_024282861.1), Oryzias latipes (XP_023811060.1), Mastacembelus armatus (XP_026162837.1), and Larimichthys polyactis. Yellow regions indicate the conserved amino acid sequences in all species, and blue regions indicate the sequences with a similarity of more than $50 \%$. Red and blue boxes represent the ATP-hydrolysis sites and microtubule-binding sites, respectively 


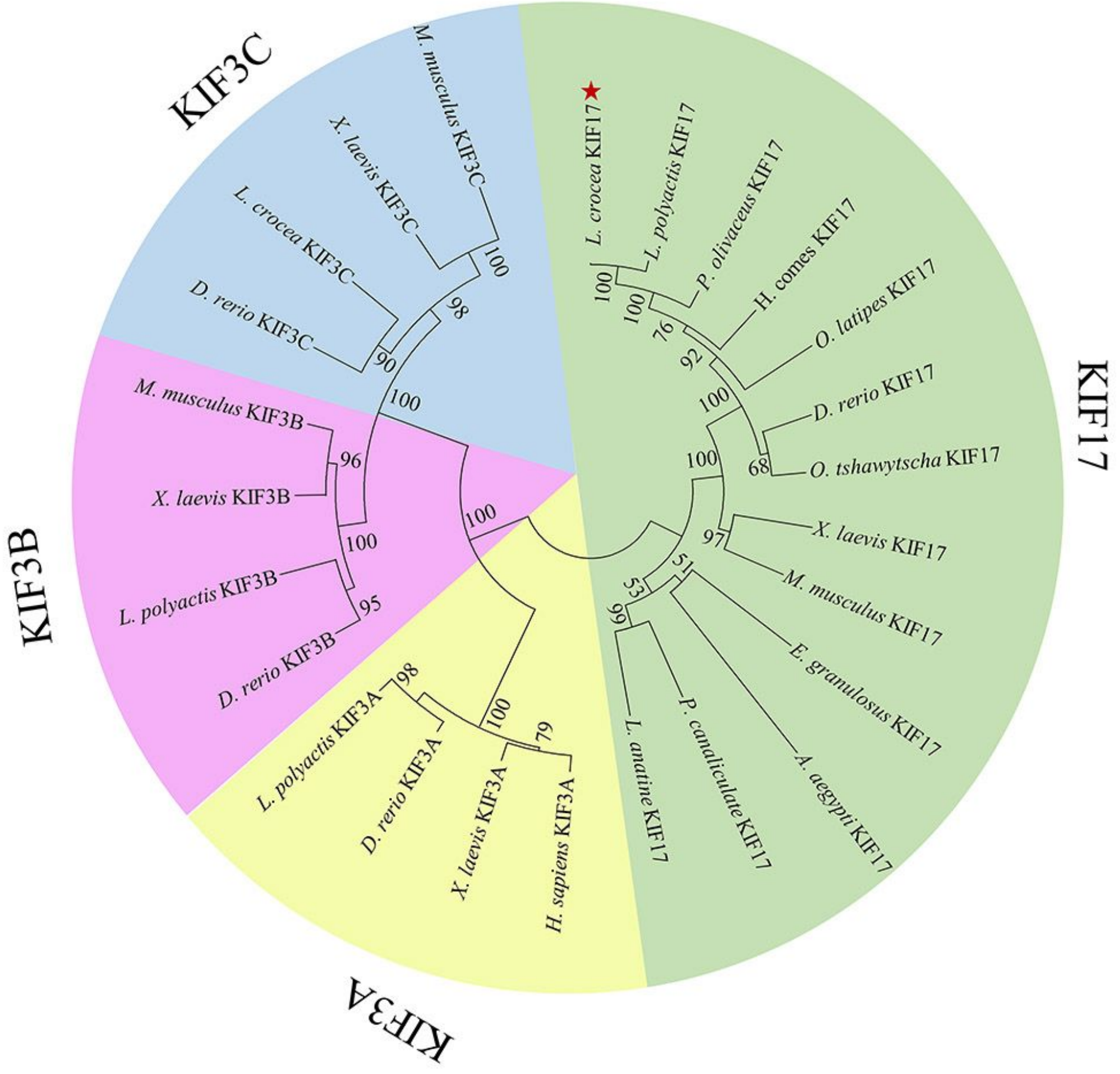

\section{Figure 4}

Phylogenetic relationship between KIF17 and other members of the kinesin-2 family. Amino acid sequences were obtained from GenBank. Homo sapiens: KIF3A (NP_001287720.1); Danio rerio: KIF3A (NP_001017604.2), KIF3B (NP_001093615.1), KIF17 (XP_001919146.1), and KIF3C (XP_002661420.3); Xenopus laevis: KIF3A (XP_018111667.1), KIF3B (XP_018092567.1), KIF17 (XP_018097355.1), and KIF3C (XP_018121538.1); Larimichthys crocea: KIF3C (XP_010738232.2); Paralichthys olivaceus: KIF17 (XP_019952108.1); Echinococcus granulosus: KIF17 (XP_024349597.1); Oryzias latipes: KIF17 (XP_023811060.1); Hippocampus comes: KIF17 (XP_019712241.1); Oncorhynchus tshawytscha: KIF17 
(XP_024282861.1); Lingula anatine: KIF17 (XP_013396997.1); Pomacea canaliculate: KIF17 (XP_025086307.1); Aedes aegypti: KIF17 (XP_021693306.1); Larimichthys polyactis: KIF3B (QBH87853.1), KIF3A (QBH87852.1), and KIF17; and Mus musculus: KIF3B (NP_032470.3), KIF17 ( NP_034753.1), and KIF3C (NP_032471.2)
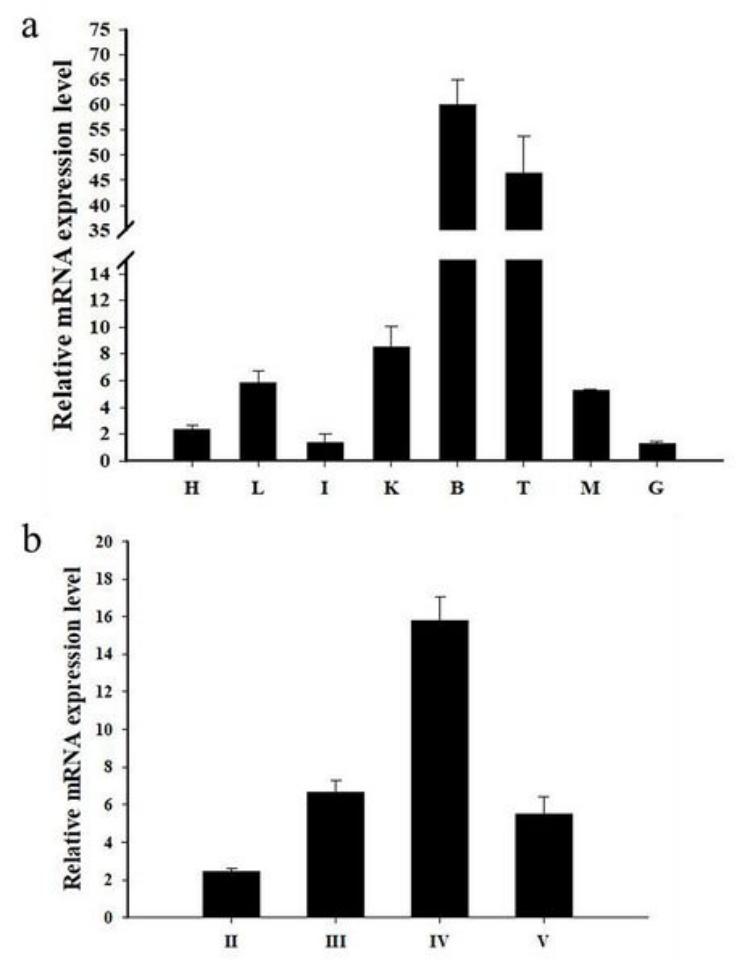
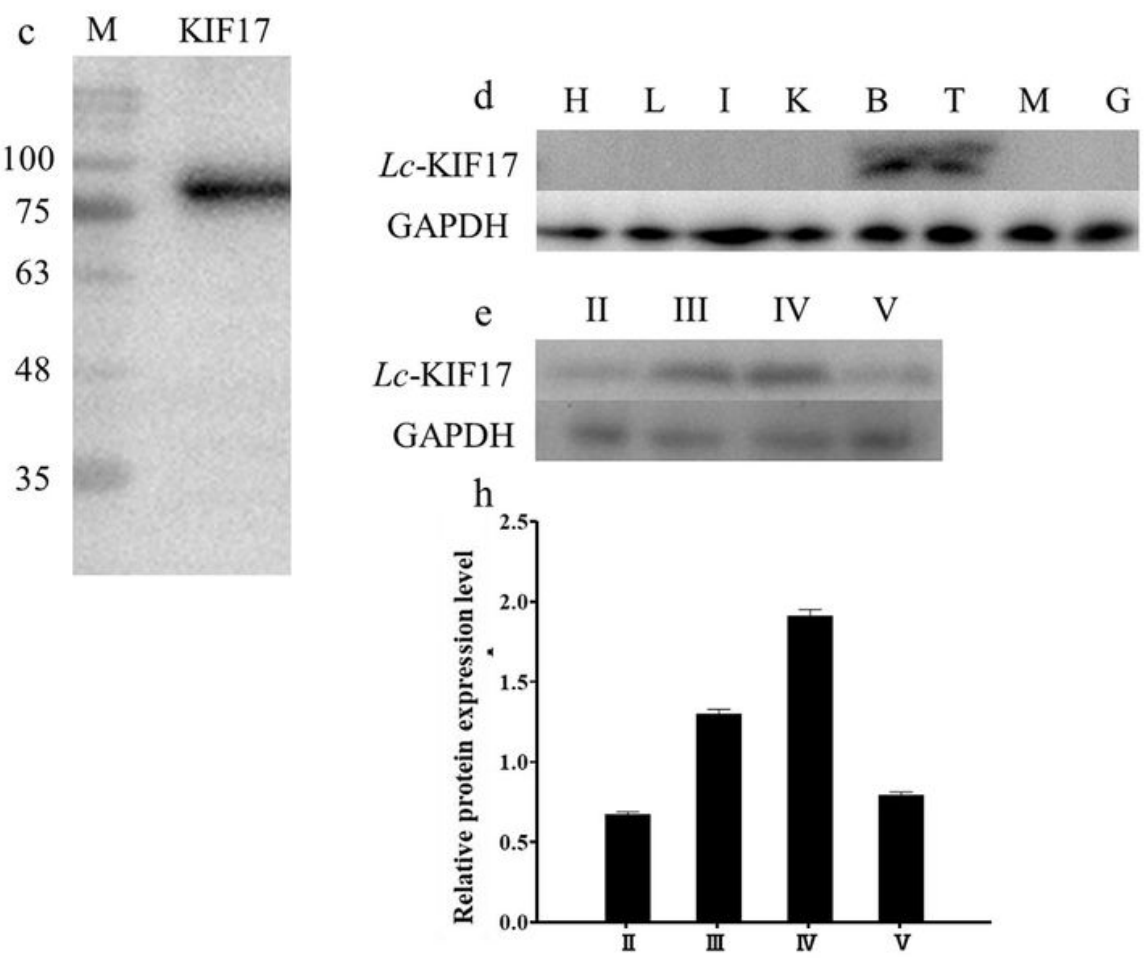

\section{Figure 5}

Tissue distribution of Lc-KIF17 at mRNA and protein levels. (a) Lc-kif17 mRNA expression in different tissues of Larimichthys crocea. (b) Lc-kif17 mRNA expression at stages II-V of testicular development. (c) Specificity assessment of mouse anti-KIF17 polyclonal antibody. A $92 \mathrm{kDa}$ protein band was detected. M: protein marker. (d) Lc-KIF17 expression in different tissues of L. crocea. (e) Lc-KIF17 expression at stages II-V of testicular development. (h) Analyses of Lc-KIF17 expression using GraphPad Prism 7. H: heart, L: liver, I: intestine K: kidney, B: brain, T: testis, M: muscle, G: gill 


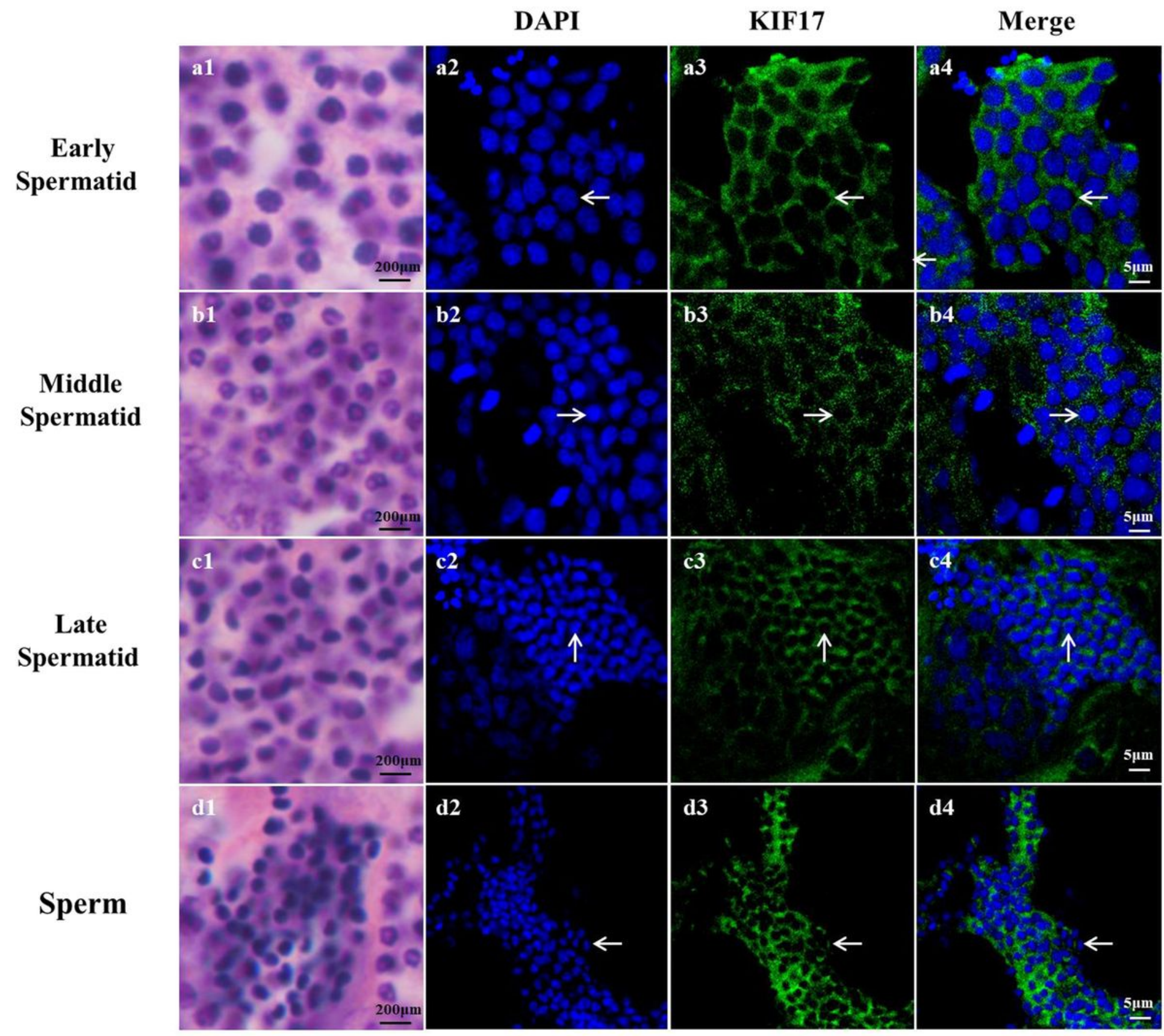

Figure 6

Spatiotemporal expression of the Lc-kif17 mRNA during Larimichthys crocea spermiogenesis. Nuclei are stained with DAPI (blue). Lc-kif17 mRNA is stained with FITC (green). (a1, b1, c1, d1) Stages II-V of testicular development in L. crocea, H\&E stain. (a2-a4) Early-stage spermiogenesis. Lc-kif17 mRNA signals are distributed in the perinuclear cytoplasm. (b2-b4) Middle-stage spermiogenesis. Lc-kif17 mRNA signals are enhanced and distributed around the nucleus. (c2-c4) Late-stage spermiogenesis. Lckif17 mRNA signals are detected on the side of the nucleus where the tail forms. (d2-d4) Mature sperm. Signals mainly appear in the midpiece 


\section{DAPI}
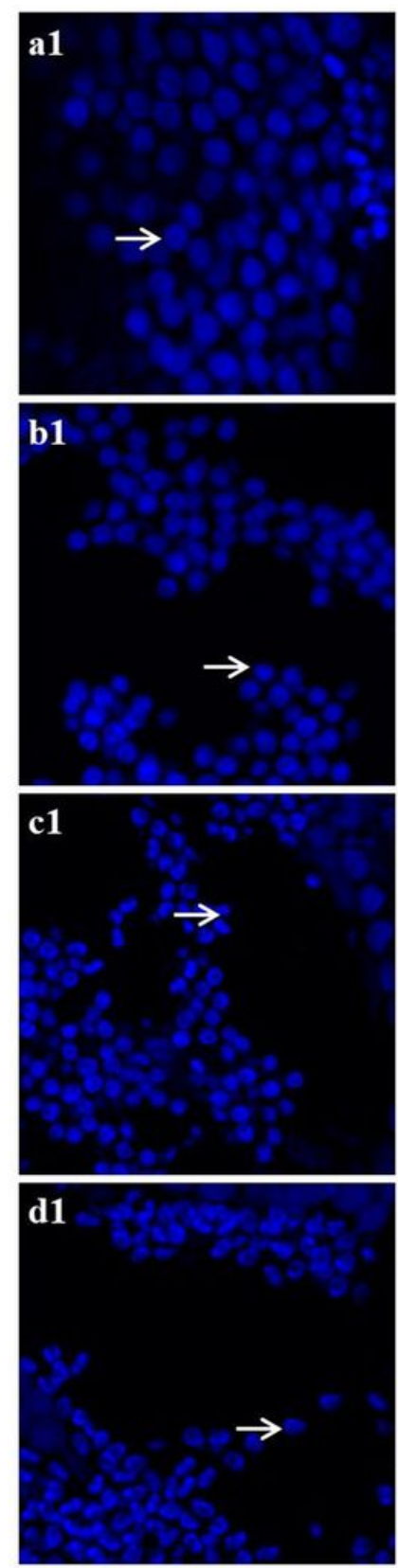

Tubulin
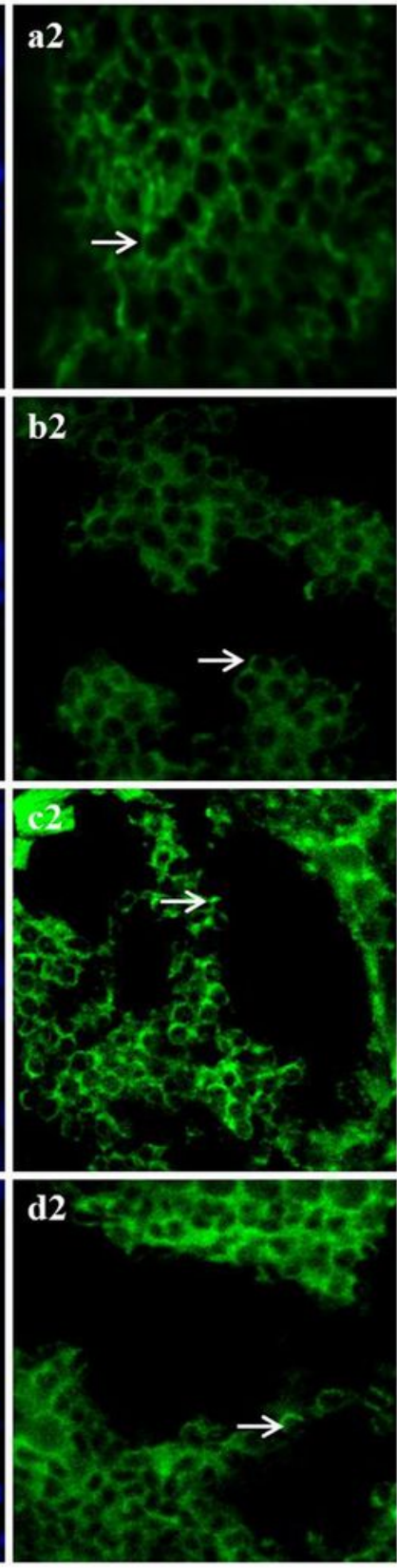

KIF17
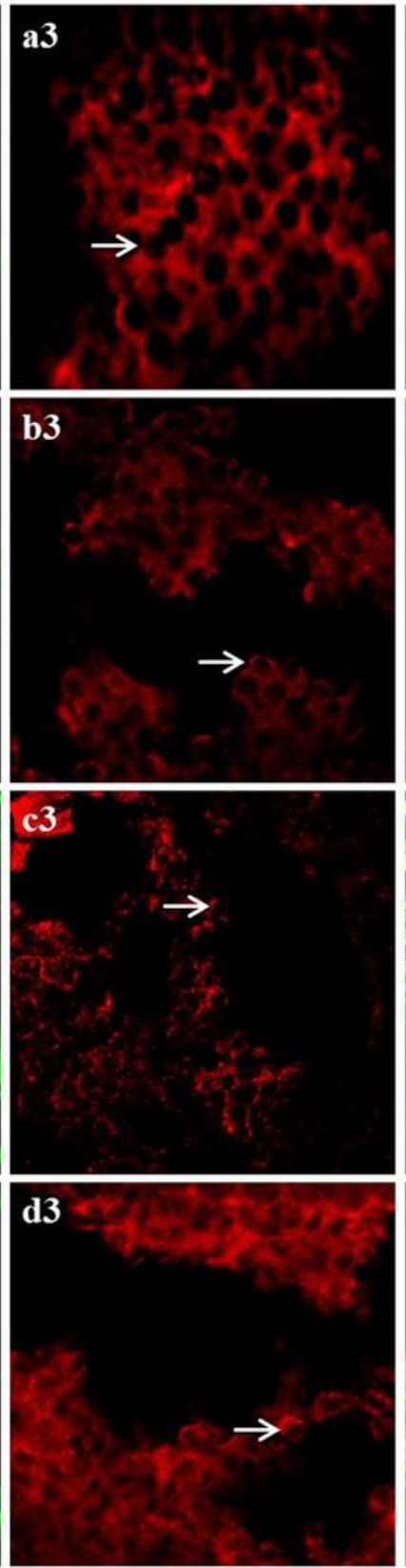

Merge
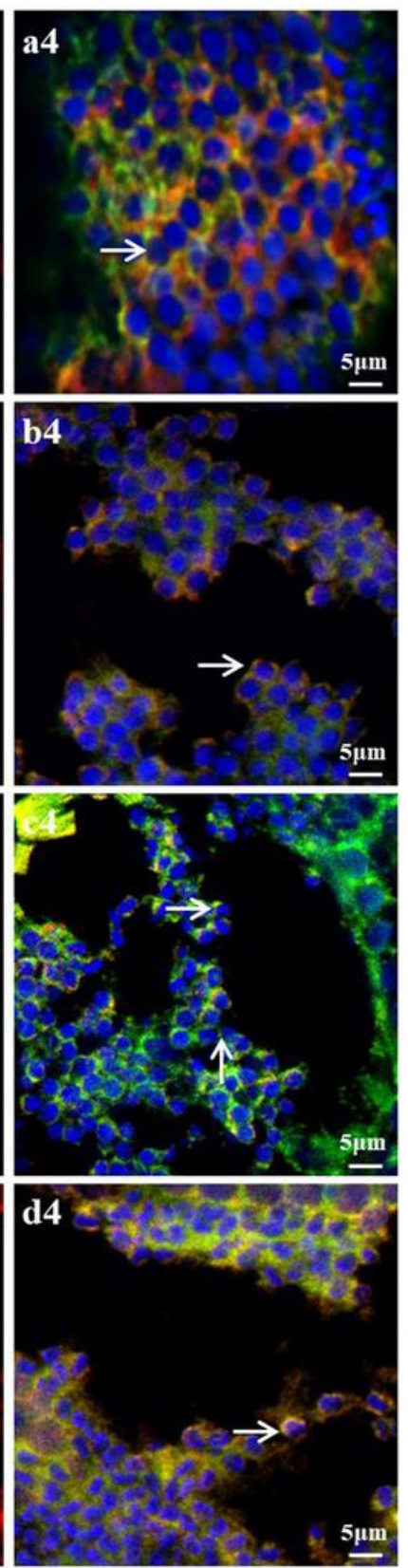

\section{Figure 7}

Immunofluorescence localization of Lc-KIF17 and tubulin during spermiogenesis. Nuclei are stained with DAPI (blue). Lc-KIF17 is stained with Alexa Fluor 555-labeled antibody (red). Microtubules are stained with Alexa Fluor 488-labeled antibody (green). Lc-KIF17 colocalizes with tubulin during Larimichthys crocea spermiogenesis. (a1-a4) Early-stage spermiogenesis. Lc-KIF17 protein signals are randomly distributed in the perinuclear cytoplasm; (b1-b4) Middle-stage spermiogenesis. Lc-KIF17 protein signals are enhanced and distributed around the nucleus. (c1-c4) Late-stage spermiogenesis. Lc-KIF17 protein signals are detected on the side of the nucleus where the tail forms. (d1-d4) Mature sperm. Signals mainly appear at the midpiece 


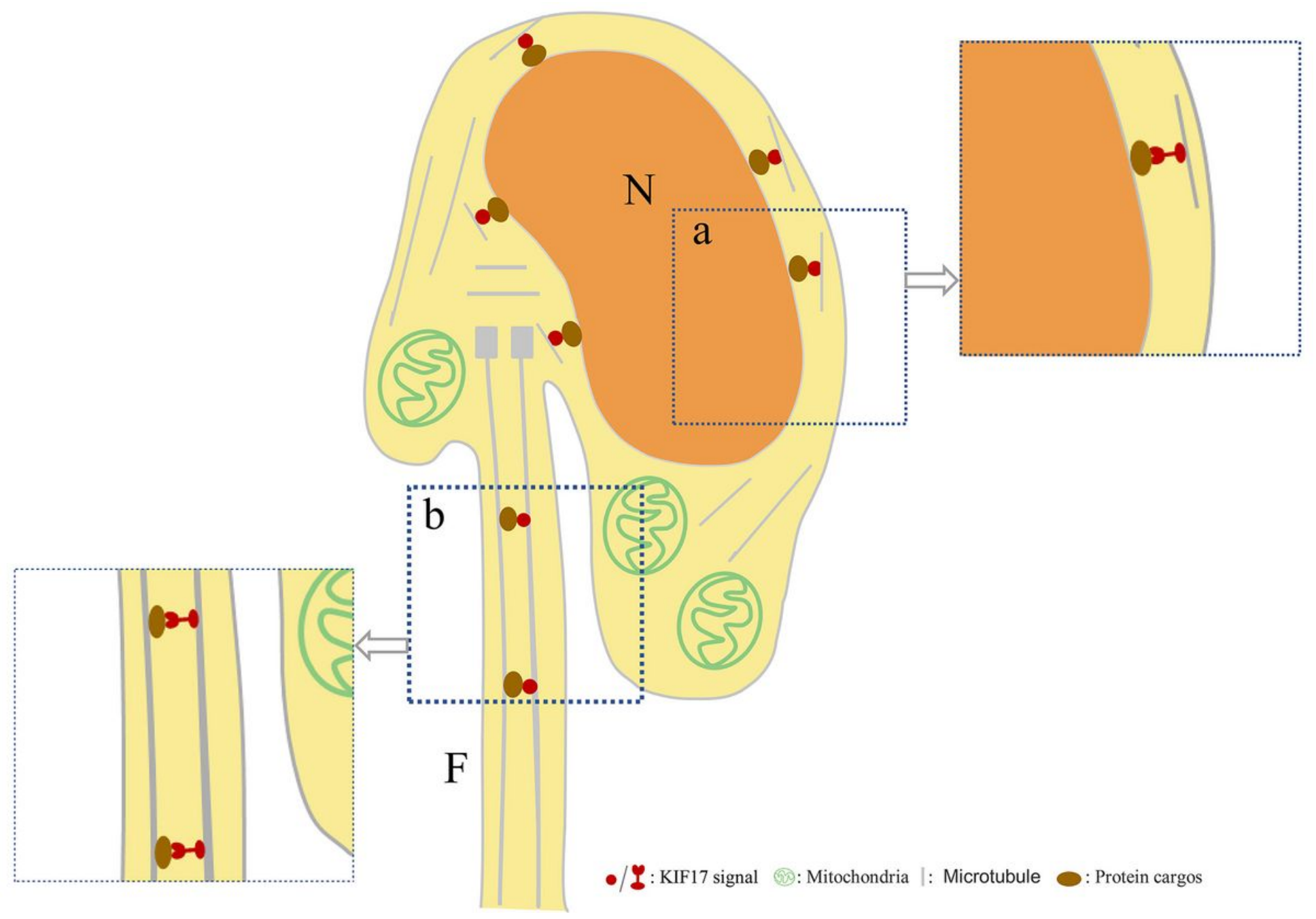

Figure 8

Predicted functional models of Lc-KIF17 during spermiogenesis. KIF17 may participate in spermatid remodeling by interacting with perinuclear microtubules during Larimichthys crocea spermiogenesis 